\title{
Linx
}

Revue des linguistes de l'université Paris X Nanterre

56 | 2007

Linguistique des genres

\section{Volochinov et Bakhtine : deux approches radicalement opposées des genres de textes et de leur statut}

Cristian Bota et Jean-Paul Bronckart

\section{OpenEdition}

Journals

Édition électronique

URL : http://journals.openedition.org/linx/360

DOI : $10.4000 / \operatorname{linx} .360$

ISSN : 2118-9692

Éditeur

Presses universitaires de Paris Nanterre

Édition imprimée

Date de publication : 1 juin 2007

Pagination : 73-89

ISSN : 0246-8743

\section{Référence électronique}

Cristian Bota et Jean-Paul Bronckart, « Volochinov et Bakhtine : deux approches radicalement opposées des genres de textes et de leur statut», Linx [En ligne], 56 | 2007, mis en ligne le 21 février 2011, consulté le 21 avril 2019. URL : http://journals.openedition.org/linx/360 ; DOI : 10.4000/linx.360 


\title{
Volochinov et Bakhtine : deux approches radicalement opposées des genres de textes et de leur statut
}

\author{
Cristian Bota, Jean-Paul Bronckart
}

\begin{abstract}
L'essai par lequel s'ouvre ce volume, et qui est le premier écrit connu de Bakhtine [...] complète et éclaire trois autres livres, parus en 1927 et 1929 sous la signature de N. Volochinov (Le Freudisme et Marxisme et philosophie du langage) et de P. Medvedev (La méthode formelle dans les sciences de la littérature), mais qui, aujourd'hui, lui sont généralement attribués : indépendamment même de la problématique qui leur est commune, le style, avec sa rigueur démonstrative, sa précision et sa vigueur imagée dans le maniement des termes abstraits, confirmerait, s'il en était besoin, la paternité de Bakhtine. Nous avons là l'exemple assez rare d'un savant acceptant l'anonymat, sacrifiant sa notoriété personnelle à la diffusion de son œuvre. (Aucouturier, 1978, pp. 10-11).
\end{abstract}

Comme les documents préparatoires à ce numéro, ces lignes de la Préface du recueil Esthétique et théorie du roman reproduisent la thèse selon laquelle Bakhtine serait le véritable auteur des ouvrages et articles qu'auraient complaisamment signés Volochinov et Medvedev. Sur la base des éléments historiques aujourd'hui disponibles (cf. Ivanova, 2003a, 2003b; Sériot, 2007), il y a lieu de rejeter définitivement cette thèse, issue de la machination conçue notamment par Ivanov $^{1}$ au début des années

\footnotetext{
${ }^{1}$ Le sémioticien Ivanov a formulé cette thèse dans deux articles publiés en 1973 et 1975 ; Bakhtine quant à lui «a laissé faire », mais s'il semble avoir confirmé oralement qu'il était effectivement l'auteur des texte signés Volochinov et Medvedev, il n’a néanmoins jamais formellement revendiqué cette
} 
1970, dans le cadre de la publication-célébration de «l'œuvre bakhtinienne ». L'analyse contrastive des écrits de Volochinov et de Bakhtine que nous proposons dans ce qui suit n'a néanmoins pas comme seul objectif de rendre à César ce qui lui revient ; elle vise aussi et surtout à montrer que les travaux de ces deux auteurs s'ancrent dans des épistémologies radicalement opposées, et que ces divergences ont des incidences majeures sur le statut qui peut être conféré au langage, aux textes et aux genres, et plus particulièrement sur le rôle que jouent ces derniers dans la constitution et le développement de l'humain.

Pour ce faire, nous retracerons d'abord les étapes majeures des parcours de Volochinov et de Bakhtine, et nous tenterons de dresser un tableau des conditions d'interaction et d'interpénétration des deux œuvres. Nous centrant ensuite sur la seule période 1920-1930, nous examinerons la teneur de la «crise» des sciences humaines alors régnante, et montrerons que les membres du prétendu "Cercle de Bakhtine », s'ils avaient en commun de rejeter les options positivistes et naturalistes, divergeaient quant à l'épistémologie qu'il convenait de leur opposer; puis nous montrerons, en nous fondant sur les écrits de l'époque, que Bakhtine avait une position clairement inspirée de la phénoménologie radicale, alors que Volochinov s'inscrivait nettement dans une perspective interactionniste sociale adossée au marxisme, très proche de celle développée par Vygotski (1927/1999). Nous analyserons enfin la reprise-transformation des thèmes majeurs de Volochinov effectuée par Bakhtine dans Les genres du discours, et soutiendrons que ce n'est qu'à la condition de leur réintégration dans l'épistémologie interactionniste de leur véritable auteur que les notions de "genres de textes », de «dialogisme» et $\mathrm{d}^{\prime}$ «attitude responsive active» se révèlent décisives pour une approche du rôle du langage dans le développement humain.

\section{Volochinov, Bakhtine et Bakhtine/Volochinov}

Nés tous deux en 1895 (le premier à Saint-Pétersbourg, le second à Orel), Volochinov et Bakhtine se rencontrent d'abord à Nevel, en 1918, au cours des réunions du cercle philosophique de M. Kagan, puis se retrouvent ensuite à Vitebsk en 1921. Volochinov y était responsable du département de musique de l'Ecole des arts théâtraux, avait recueilli Bakhtine dans sa maison et, avec l'appui de Medvedev, lui avait trouvé des tâches d'enseignement dans diverses institutions : à cette époque, si « cercle » il y avait, c'était manifestement celui de Volochinov/Medvedev, et Bakhtine n'en était qu'un membre plutôt marginal, en raison de ses options phénoménologiques et de ses préoccupations religieuses (cf. Todorov, 1981, p. 14). En 1922, Volochinov

paternité (cf. Todorov, 1981, pp. 16-24 ; Depretto, 1997, p. 170). Bien qu'aucune réelle preuve n'ait été fournie à son appui, bien que les raisons invoquées par Bakhtine pour justifier cette substitution n'aient aucune crédibilité (« ses amis avaient besoin de publier!», cf. Botcharov 1997, p. 183), bien que l'on ne puisse comprendre pourquoi un auteur se déclarant anti-marxiste aurait publié des textes de cette teneur, et surtout bien qu'une analyse contrastive minimale des textes des auteurs concernés suffise à en démontrer l'inanité, cette thèse a été acceptée et diffusée, par Jakobson d'abord (cf. 1977), puis par l'ensemble des préfaciers ou commentateurs des textes «bakhtiniens », nombre d'entre eux n'hésitant pas d'ailleurs à apporter une touche personnelle à la confection de ce « conte merveilleux ». 
part pour Petrograd, où il poursuit des études d'ethnolinguistique à la Faculté des sciences sociales, puis élabore sa thèse de doctorat à l'Institut d'étude comparative des littératures et des langues occidentales et orientales [thèse soutenue en 1926 et dont Marxisme et philosophie du langage (V52) constitue une version abrégée]. En 1930, il est nommé maître de conférences à l'Institut pédagogique Hertzen, puis professeur dans une institution de formation continue ; il meurt prématurément de tuberculose en 1936. Bakhtine quant à lui quitte Vitebsk en 1924 pour Leningrad ${ }^{3}$ où, ayant obtenu un poste de collaborateur à l'Institut d'Histoire de l'Art, il reprend contact avec Volochinov et Medvedev, mais fréquente surtout les cercles religieux ("Résurrection » et «Confrérie de Saint-Séraphim»); arrêté pour cette raison en 1928, il est ensuite condamné aux camps, puis finalement « relégué » à Koustantaï (Kazakhstan) en 1930. Après avoir travaillé six ans dans cette ville comme économiste comptable, grâce à l'intervention de Medvedev, il est affecté en 1936 à l'Institut pédagogique de Mordovie (Saransk), qu'il quitte cependant rapidement pour s'installer à Savelovo, près de Moscou. En 1945, il revient à Saransk et y soutient sa thèse l'année suivante (B8 L'auvre de François Rabelais) puis, cet institut devenu université en 1957, il y dirige la section de littérature russe et étrangère jusqu'à sa retraite en 1961. En 1969, il s'installe à Moscou, où il résidera jusqu'à sa mort en 1975.

Les écrits connus de Volochinov (deux ouvrages et sept articles) ont tous été publiés à l'époque de leur rédaction, entre 1925 et 1930. L’auteur était alors reconnu dans le champ des études linguistiques/littéraires, ce qui lui valut d'être appelé à collaborer avec Jakubinski à la revue Literatournaïa outchioba que dirigeait M. Gorki. L'ensemble de ses textes constituent un corpus cohérent, témoignent d'une ferme orientation sociologique et marxienne et sont rédigés en un style rugueux bien éloigné de celui des textes proprement bakhtiniens. Quant à Bakhtine, son activité d'écriture s'est déployée en quatre phases. De 1920 à 1929, il rédige d'une part La philosophie de l'acte (B1), texte d'inspiration à la fois phénoménologique et religieuse, d'autre part deux autres manuscrits (B2, B3) et le livre sur Dostoievski (B4), textes de critique littéraire s'inscrivant en opposition à l'approche des formalistes. De 1936 à 1941, il rédige une deuxième série de manuscrits témoignant de cette même double orientation (B5, B6, B7) et il publie sa thèse sur Rabelais (B8). De 1946 à 1961, il rédige ses deux manuscrits célèbres sur Les genres du discours et Le problème du texte (B9 et B10). De cette période à sa mort, il retravaille essentiellement ses manuscrits des périodes précédentes, en vue de la publication des recueils des années 1970 (traduits en français en 1978 et 1984).

Les interactions effectives des deux œuvres se présentent dès lors comme suit. De 1920 à 1930, les travaux des deux auteurs sont radicalement différents, dans leur teneur théorique aussi bien que dans leurs sources de référence et dans leurs modalités de composition. Mais c'est dans les textes de Volochinov que sont introduits et développés ${ }^{4}$ les thèmes fondamentaux des «types et formes de discours», de

\footnotetext{
${ }^{2}$ Les textes des deux auteurs que nous exploiterons sont désignés par les abréviations V1 à V9 pour Volochinov, et B1 à B10 pour Bakhtine; cf. bibliographie.

${ }^{3}$ Nouveau nom donné cette année-là à la ville de Petrograd, ex- et future Saint-Pétersbourg.

${ }^{4}$ Dans son Dostö̈evski (B4), Bakhtine évoque certes le dialogisme, mais sans développements dépassant l'approche issue de Jakubinski; et il traite aussi des genres, mais dans la perspective
} 
l'énonciation comme interaction sociale, ou encore du dialogisme et de l'attitude responsive active (cf. 4, infra). Après la disparition de Volochinov, Bakhtine reprend alors ces thèmes à son compte, de plus en plus nettement, et les reformule dans sa propre perspective (toujours phénoménologique), mais sans jamais citer Volochinov (ni Medvedev). "Mon Dieu, combien de fois n'ai-je pas recopié ce livre», aurait spontanément déclaré Madame Bakhtine à un critique soviétique lui montrant Marxisme et philosopbie du langage (cf. Todorov, 1981, p. 16). Dès lors qu'il est définitivement établi que Volochinov est le seul auteur de cet ouvrage, que Bakhtine ne pouvait évidemment ignorer cette réalité, et plus généralement qu'il connaissait l'ensemble des travaux de celui qui fut si longtemps son «ami », cette anecdote mentionnée à l'appui de la thèse d'Ivanov prend alors une toute autre signification : dès 1936, et surtout après 1946, Bakhtine a en réalité exploité sans vergogne les écrits de Volochinov; ce véritable pillage risquait d'être identifié et dénoncé; et c'est très vraisemblablement pour faire pièce à ce danger qu'a alors été construite et diffusée la fable selon laquelle Bakhtine était en fait «l'auteur masqué » des écrits signés Volochinov et Medvedev!

\section{Langage, littérature et « crise » des sciences humaines}

L'exceptionnelle profondeur des approches du langage et de la littérature qui se sont développées en Russie (puis en Union soviétique) dans le premier tiers du XXe tient fondamentalement à ce que celles-ci reposaient sur une connaissance détaillée des apports des autres sciences humaines, et qu'elles s'articulaient à leur questionnement général : ces démarches visaient moins à l'élaboration d'une théorie du langage en tant que tel, ou de la littérature comme phénomène autonome, qu'à la compréhension du rôle que jouaient les pratiques verbales, qu'elles soient ordinaires ou littéraires, dans le fonctionnement psychique et dans l'organisation sociale des humains. Les écrits et enseignements de Potebnya ${ }^{5}$ (1913) notamment étaient centrés sur la différence entre langue de la poésie et langue de la prose, ainsi que sur la question des relations entre mots et unités de pensée, et ceux de Jakubinski ${ }^{6}$ (1923) sur les dimensions sociales et psychologiques de l'interaction verbale. Comme en attestent leurs écrits des années 1920-1930, Volochinov et Bakhtine s'inscrivaient dans cette perspective générale, l'un et l'autre débattant longuement des diverses positions émanant de la philosophie et des sciences humaines ${ }^{7}$, en particulier sur la question du statut de la conscience et sur celle des rapports entre cette dernière et les œuvres culturelles.

L'ampleur et la complexité des débats sur ces thèmes avaient fini par engendrer un diagnostic global de «crise » des sciences humaines (et plus particulièrement de la

traditionnelle centrée sur la littérature (et non sur les genres ordinaires dans leurs rapports aux formes de l'activité humaine).

${ }^{5}$ Vygotski fait fréquemment référence aux travaux de Potebnya, et les ouvrages majeurs de ces deux auteurs ont exactement le même titre : La pensée et la langue.

${ }^{6}$ Formé à l'école de Kazan, Jakubinski a élaboré la première approche du « dialogisme », dont s'est notamment inspiré Volochinov (cf. Ivanova, 2003b).

${ }^{7}$ Comme en attestent ses écrits sur le freudisme (cf. V1 et V3) Volochinov avait une connaissance remarquable de l'ensemble des travaux de la psychologie de son époque. 
psychologie : cf. notamment Bühler, 1927 ; Politzer, 1928 ; Vygotski, 1927/1999), crise dont les causes et les enjeux peuvent être résumés comme suit.

En créant le premier laboratoire de psychologie expérimentale, Wundt avait posé que les processus mentaux et la conscience constituaient les objets majeurs de cette discipline, et qu'ils devaient être abordés en une démarche objective analogue à celle des sciences naturelles (cf. 1874-1875). Mais ce «pari» n'a pu être tenu, les expérimentations réalisées ne donnant accès qu'aux processus élémentaires de perception et d'association, et ne permettant pas de mettre en évidence les modalités de fonctionnement des processus de pensée et de conscience. L'œuvre de Wundt s'est alors poursuivie de manière dichotomique, les processus psychologiques inférieurs étant abordés dans le cadre d'expériences de laboratoire, et les processus supérieurs étant traités par une tout autre méthode, l'observation comparative des phénomènes sociaux et culturels (démarche ayant donné lieu aux dix volumes de sa Völkerpsychologie, 1900-1920).

Cette bipartition issue de l'œuvre de Wundt a donné naissance, chez ses successeurs, à la constitution de deux ${ }^{8}$ camps radicalement opposés. Illustré notamment par la réflexologie de Pavlov et de Bekhterev, puis par le behaviorisme de Watson, un premier courant a considéré que la psychologie ne pouvait se donner comme objets que les phénomènes accessibles aux démarches expérimentales: il s'agissait de ne prendre en compte que les «observables » (comportements et facteurs du milieu), de procéder à une description systématique de leurs interactions, et de mettre ainsi en évidence des relations de dépendance (stimulus-réponse) et/ou des lois d'apprentissage. Dans cette perspective, la conscience et les phénomènes psychiques (intentions, idées, sentiments, etc.) étaient considérés comme des appellations préscientifiques obscurcissant la compréhension des mécanismes ayant cours à l'intérieur des organismes, mécanismes dont l'étude devait permettre, à terme, de statuer sur ces phénomènes externes complexes que constituent le langage et les diverses constructions socioculturelles. Un second courant a considéré qu'au contraire les processus de conscience d'un côté, les œuvres culturelles d'un autre, constituaient les objets spécifiques des sciences de l'humain, et qu'il fallait dès lors les aborder de front. Mais cette résistance au positivisme s'est elle-même développée en deux tendances radicalement opposées.

Le premier courant était directement inspiré de la philosophie de Brentano (1874/1944), selon laquelle les phénomènes psychiques conscients ne sont attestables que sous la forme d'un vécu intérieur du sujet, à la fois indubitable (cf. le cogito cartésien) et immédiatement accessible, ce qui implique qu'ils ne peuvent être utilement étudiés ni dans leurs rapports aux processus inférieurs, ni en tant que construits sociohistoriques : ils doivent au contraire faire l'objet d'une démarche de description synchronique de tout ce qui est effectivement ressenti ou vécu. Ce positionnement a d'abord donné naissance au courant de psychologie empirique ou descriptive, ensuite à la psychologie de l'acte intentionnel de Stumpf et de l'école de Würzburg : utilisant la méthode d'introspection expérimentale systématique, ces chercheurs ont tenté de montrer que les

\footnotetext{
${ }^{8}$ Cette opposition entre deux camps est évidemment trop schématique, et ne prend pas en compte les positions intermédiaires ou «conciliatrices» illustrées notamment par les travaux de James, de Stern ou de l'école de la Gestalt (pour une approche plus détaillée, cf. Bronckart \& Friedrich, 1999).
} 
processus psychologiques supérieurs, d'une part se constituent dans une indépendance relative à l'égard des sensations et des associations élémentaires, d'autre part se caractérisent par une orientation finalisée, ou encore par l'existence de « schèmes de la pensée ». Par ailleurs, dans le champ proprement philosophique, cette position a été radicalisée par la phénoménologie de Husserl (1931), qui soutient qu'au niveau des processus psychiques conscients, dimensions ontologiques et phénoménologiques coïncident nécessairement ( il n’y a d'être que dans le phénomène ») ; ce qui revient à récuser toute possibilité d'une approche expérimentale de la conscience, dont la problématique est finalement renvoyée à un idéalisme transcendantal, au-delà de toute distinction sujet-monde (ou intérieur-extérieur).

Issu du behaviorisme social de Dewey (1925) et de Mead (1934/2006) et surtout de l'approche historico-culturelle de Vygotski, et qualifié aujourd'hui d'interactionnisme social, le second courant était clairement inspiré du monisme spinozien ainsi que de la philosophie marxiste. Son principe premier était que l'objet d'une science humaine n'est pas le rapport qui s'instaure entre un sujet pensant et le monde, mais bien la praxis collective, ou encore "l'activité humaine elle-même, en tant qu'activité objective 》 (Marx, $1845 / 1951$, p. 63) ; et sa thèse centrale était que les capacités de pensée active des humains ne découlent pas directement des propriétés de leurs corps ou de leurs comportements objectifs, mais bien plutôt de la réintégration, en chaque organisme, des propriétés de la vie sociale objective, dans ses aspects de création d'instruments et de coopération par le travail et le langage (cf. Engels, 1925/1975). Dans cette perspective, le langage était conçu comme un usage interactif organisé en discours, dont les unités (ou signes) ont la propriété de fixer les représentations du monde dans le mouvement même où elles les rendent partageables ou collectives, et celle de conserver ces représentations signifiantes au titre de «témoins de l'histoire ». Et dans ce cadre, la démarche développementale de Vygotski a eu pour objectif de démontrer que l'appropriation et l'intériorisation des signes étaient constitutives de la pensée consciente proprement humaine, et qu'en conséquence, ces signes étant toujours déjà sociaux, la conscience pouvait être définie comme « un contact social avec soi-même » (1925/1994, p. 46).

\section{Les orientations épistémologiques de Bakhtine et de Volochinov}

S'ils s'opposaient l'un et l'autre au positivisme, Bakhtine s'inscrivait clairement dans le courant phénoménologique, et Volochinov tout aussi clairement dans le courant opposé de l'interactionnisme social comme nous le montrerons maintenant en comparant la teneur de La philosophie de l'acte ${ }^{9}$ aux écrits de Volochinov.

\footnotetext{
${ }^{9}$ Retrouvé dans les archives de Bakhtine après sa mort, et n'ayant pas, semble-t-il, été "re-travaille" ensuite, ce manuscrit traduit dès lors la position effective de l'auteur aux débuts des années 1920. Il ne constitue qu'une partie d'un projet qui devait comprendre trois autres volets, centrés sur l'éthique de la création artistique, l'éthique politique et l'éthique religieuse.
} 


\subsection{Bakbtine, ou le monde vécu comme unique objet d'étude}

La philosophie de l'acte présente un ambitieux projet philosophique, d'ordre à la fois moral et phénoménologique, fondé sur le constat de l'inaptitude des démarches scientifiques et philosophiques à rendre compte du monde vécu, ou monde de la vie, en tant que réalité première (et ultime) de l'existence humaine :

Pour ma conscience participante et exigeante, il est clair que le monde de la philosophie contemporaine, le monde théorique et théorisé de la culture en un certain sens est réel, qu'il possède une validité, mais il est également clair pour elle que ce monde n'est pas le monde singulier dans lequel, précisément, je vis et dans lequel mon acte s'accomplit de façon responsable. Et ces mondes sont sans communication possible [...] la philosophie contemporaine ne donne pas de principe pour cette intégration [...] (B1, pp. 42-43).

L'objet général que s'y donne l'auteur est la "participation singulière à l'être » ou le vécu irréductible et concret du sujet, et pour lui l'élucidation de ce vécu participatif (appelé successivement «acte », « vie-acte », « l'être-événement un et singulier », etc.) ne peut s'effectuer que par le biais d'une description phénoménologique : « la description expérimentale du monde de la vie-acte singulière du dedans de l'acte [...] serait un autorapport/confession, individuel et singulier» (p. 84). Bien que les réflexions proposées dans cette mise en place d'une philosophie de l'acte soient évidemment plus complexes, nous en retiendrons trois aspects centraux, ayant trait aux fondements mêmes de la démarche et aux éléments qu'elle délimite.

Le premier concerne la notion centrale d'acte, dont l'acception dans ce texte n'est ni praxéologique, ni véritablement ontologique (l'acte au sens aristotélicien), mais renvoie exclusivement au terrain d'une conscience entendue comme être spirituel autonome et responsable de ses agissements. Ce faisant, l'auteur procède à une double réduction de ce concept, consistant à en éliminer toute dimension ontologique ou sociohistorique, et à le rattacher exclusivement au domaine du vécu. Plus précisément, la dimension ontologique est réduite au seul domaine des valeurs (au monde de la culture), alors que la dimension axiologique est rabattue sur le seul vécu singulier: ontologie et axiologie ne sont en fait « réelles » qu'au sein du sujet:

[la] reconnaissance de ma propre singularité comme inessentielle pour la conception de l'être, inéluctablement entraîne aussi à sa suite la perte de la singularité de l'être, et nous aboutirons à la conception d'un être seulement possible, et non pas de l'être singulier effectif qui existe, inévitablement réel. (B1, p. 36).

Dans la mesure où je vis réellement un objet, même si je ne le vis que par la pensée, il devient composante changeante de l'événement en cours qu'est mon vécu (pensée) de cet objet $[\ldots]$ Ou, plus précisément, il m'est donné dans une certaine unité événementielle, dans laquelle les composantes [...] de l'être et du devoir, de l'être et de la valeur sont inséparables. Toutes ces catégories abstraites sont là les composantes d'un certain tout vivant, concret, palpable, singulier : d'un événement. (B1, p. 57). 
Le statut de ce vécu est en outre d'ordre moral, dans la mesure où il se déploie sous l'effet de la responsabilité (ou de la conscience du devoir), qui est la source de tous les actes du sujet ainsi que le garant des valeurs:

Le devoir est une catégorie originale de l'action ou de l'acte (et tout est mon acte, même la pensée et le sentiment), est une certaine attitude de la conscience dont nous nous attacherons à révéler la structure phénoménologiquement. (B1, p. 22).

En bref, l'auteur se propose donc de développer une phénoménologie dans laquelle les propriétés d'une mystérieuse intentionnalité originaire sont transférées au devoir, à la responsabilité, comme propriété intrinsèque et définitoire du sujet.

Le deuxième aspect a trait aux composantes de l'acte que constituent les participants au «monde » (le moi et l'autre) : si l'auteur affirme qu'en raison de leur radicale singularité, leurs vécus respectifs sont incommunicables, il postule néanmoins in extremis l'existence d'une force cohésive de ces vécus, en une argumentation se présentant comme suit. Le sujet constitue le donné primaire et irréductible du « monde réel de l'acte» : il est une réalité auto-engendrée autour de laquelle s'organise le monde extérieur des valeurs :

pour ma conscience agissante participante, [le monde] est disposé [...] autour de moi comme seul centre d'émergence de mon acte : je tombe sur ce monde dans la mesure où j'émerge de moi-même [...] (B1, p. 88).

Ce sujet participe au monde en affirmant des valeurs propres à sa conscience singulière :

La vie connait deux centres de valeurs différents dans leur principe même, mais corrélés entre eux : moi et l'autre, et c'est autour de ces centres que sont réparties et disposées toutes les composantes concrètes de l'être. (B1, p. 110).

Saisies indépendamment de ces «centres», les valeurs existantes (scientifiques, esthétiques, éthiques), voire l'espace et le temps, n'ont aucune réalité (B1, p. 85). Les valeurs nouvelles affirmées par le sujet sont incompatibles avec les précédentes et entrent donc en conflit avec elles; et c'est ce conflit qui constitue l'« événement» ou le «réel»: « une multitude de mondes personnels d'une valeur non reproductible (...) crée pour la première fois l'événement un» (B1, pp. 74-75). Enfin, ce conflit ("hétéronymie du vécu concret de soi et du vécu de l'autre ») doit se soumettre à un principe de corrélation, qui garantit la communicabilité des vécus axiologiques et, ultimement, leur transformation en de nouvelles valeurs «objectives». Et pour l'auteur, cette corrélation de la multiplicité des valeurs est réalisée sous l'effet de la responsabilité ${ }^{10}$ :

Chacun a raison à sa propre place, a raison non pas subjectivement, mais d'une manière responsable. (B1, p. 75).

\footnotetext{
10 En ce qui concerne les valeurs esthétiques, la corrélation des valeurs est réalisée par l'« amour esthétique objectif » de l'auteur (B1, p. 95).
} 
Le troisième aspect concerne le langage, dont le rôle est quasiment réduit à néant: Bakhtine n'évoque que le mot, dont la fonction est entièrement dévolue à l'expression immédiate du vécu participatif, et dont la signification ne procède (en l'absence de toute perspective sociale) que d'une seule et même instance morale autonome :

Historiquement le langage s'est développé au service de la pensée participante et de l'acte (...). L'expression de l'acte à partir du dedans, et l'expression de l'êtreévénement singulier dans lequel cet acte est accompli requièrent toute la plénitude du mot : son aspect de contenu de sens (le mot-concept), son aspect palpable-expressif (le mot-image) et son aspect émotif-volitif (l’intonation du mot) dans leur unité. Et dans toutes ces composantes, le mot plein et un peut être signifiant de façon responsable - peut être la vérité - et non pas quelque chose de subjectivement fortuit. (p. 56).

C'est en définitive cette instance de responsabilité morale qui constitue le garant de la signification du mot et, par là-même, le garant de la vérité de l'expression.

Si elle demeure sommaire, cette analyse suffit à montrer que l'écrit-princeps de Bakhtine s'inscrit dans un cadre dualiste radical, attribuant à l'humain une essence universelle innée (la pensée responsable) radicalement disjointe du reste de la Nature. Cette adhésion à la thèse de l'existence d'une substance spirituelle/morale autonome entraîne une centration exclusive sur le sujet et ses capacités intrinsèques, ce qui débouche sur une conception particulièrement solipsiste, dans laquelle l'ensemble des dimensions historiques, sociales et signifiantes de l'activité humaine sont de fait considérées comme des émanations de la conscience morale du sujet.

\subsection{Volochinov, ou l'étude de la psychologie du corps social}

$\mathrm{Au}$ fond, la philosophie de notre temps n'a qu'une idée, qui est d'édifier un monde audelà du social et de l'bistorique. (V3, p. 189)

$\mathrm{Si}$, comme Bakhtine, il critiquait la philosophie de son époque aussi bien que le positivisme des courants dominants de la psychologie, Volochinov ancrait cependant résolument sa démarche dans le cadre moniste matérialiste, et son objectif majeur était de mettre en œuvre une méthodologie générale qui, partant de l'analyse de ces concrétisations de la «psychologie ${ }^{11}$ du corps social» que constituent les interactions verbales, permettrait de démontrer le statut ontologiquement matériel de la pensée consciente bumaine:

Ce qu'on appelle la psychologie du corps social et qui constitue $[\ldots]$ une sorte de maillon intermédiaire entre la structure socio-politique et l'idéologie au sens étroit du terme (la science, l'art, etc.) se réalise, se matérialise sous forme d'interaction verbale. [...] La psychologie du corps social ne se situe pas quelque part à l'intérieur (dans les

\footnotetext{
${ }^{11}$ Dans ce type d'occurrence, le terme de «psychologie » ne désigne pas la discipline éponyme, mais vise à souligner que la collectivité constitue le lieu d'ancrage premier des phénomènes psychiques. Ce qui revient à affirmer que les interactions verbales constituent les processus effectifs de constitution des représentations collectives qu'avait magistralement analysées Durkheim (1898/1951).
} 
Cristian Bota, Jean-Paul Bronckart

«âmes» des individus en situation de communication), elle est au contraire entièrement extériorisée : dans le mot, dans le geste, dans l'acte. (V5, p. 38)

Et c'est parce que le psychique constitue l'une des propriétés de la matière organisée qu'on ne saurait en faire un principe particulier d'explication opposable au matériel. Ce qu'il faut, au contraire, c'est se placer résolument sur le terrain de l'expérience matérielle externe et, à partir de là, montrer quel type d'organisation et quel degré de complexité de la matière déterminent l'apparition de cette nouvelle qualité, de cette nouvelle propriété de la matière elle-même, qu'est le psychique. (V3, pp. 102-103)

Cette méthodologie devait en conséquence s’opérationnaliser en un programme « descendant », qui prendrait d'abord en compte les formes d'organisation collective de toute communauté humaine, puis les types de communication sociale rendus possibles par cette organisation, ensuite l'interaction verbale dans ses propriétés concrètes, et enfin les énoncés verbaux organisés en textes (cf. V5, p. 137 ; V7, p. 289) ; programme que Bakhtine n'a jamais évoqué ${ }^{12}$, et moins encore mis en œuvre.

Dans cette perspective, Volochinov s'est livré à une critique sans concession des phénoménologues inspirés de Brentano (et donc à une critique de la position de Bakhtine) :

A côté du psychisme individuel et de la conscience subjective individuelle ils font une place à la «conscience globale», la «conscience transcendantale», le «sujet purement gnoséologique », etc. C'est dans ce contexte transcendantal qu'ils localisent le phénomène idéologique, par opposition à la fonction psychique individuelle. [...] Pendant ce temps, l'idéologie [...] n'a plus sa place nulle part et se trouve contrainte d'émigrer de la réalité vers les hauteurs transcendantales ou même carrément transcendantes. (V5, pp. 54-55)

En dehors de son objectivation, de sa réalisation dans un matériau déterminé (le geste, la parole, le cri), la conscience est une fiction. Ce n'est qu'une construction idéologique incorrecte, créée sans tenir compte des données concrètes de l'expression sociale. Mais en tant qu'expression matérielle structurée [...], la conscience constitue un fait objectif et une force sociale immense. (V5, p. 129)

Sa position propre était que les significations construites dans l'activité collective se cristallisent dans les signes, et qu'il y a lieu en conséquence d'analyser les valeurs prises par les signes dans les différentes formes d'interactions verbales (ou formes de discours), pour comprendre la nature même des ingrédients constitutifs de la conscience :

\footnotetext{
12 Étant donné son adhésion à la thèse de l'omnipotence du sujet, Bakhtine ne pouvait évidemment adhérer à un tel projet; mais il se trouvait même dans l'incapacité de proposer une véritable articulation entre les résultats de ses propres analyses littéraires et son cadre phénoménologique radical, ce qui explique sans doute son absence de publication avant le Dostö̈evski (ouvrage qui reste de fait obéré par cette incohérence, comme Kristeva l'a en partie relevé - cf. 1970, p. 14).
} 
La conscience ne peut pas dériver directement de la nature comme a tenté et tente encore de le montrer le matérialisme mécaniste naif et la psychologie contemporaine [...]. L'idéologie ne peut pas dériver de la conscience, comme prétendent le faire croire l'idéalisme et le positivisme psychologiste. La conscience prend forme et existence dans les signes créées par un groupe organisé au cours de ses relations sociales. La conscience individuelle se nourrit de signes, elle y trouve la matière de son développement, elle reflète leur logique et leurs lois. [...] Si nous privons la conscience de son contenu sémiotique et idéologique, il n’en reste rien. (V5, p. 30)

Et cette position débouchait sur une approche des conditions de constitution et de développement du psychisme quasi identique à celle que développait simultanément Vygotski, et qui y ajoute même une dimension dialectique fort bienvenue ${ }^{13}$ :

La psychologie doit s'appuyer sur la science des idéologies. Le mot a dû, à l'origine, naître et se développer au cours du processus de socialisation des individus, pour être ensuite intégré à l'organisme individuel et devenir parole intérieure. Pourtant, le psychologisme a raison également : il n'y a pas de signe extérieur sans signe intérieur. Le signe extérieur incapable d'entrer dans le contexte des signes intérieurs, c'est-àdire d'être compris et éprouvé, cesse d'être un signe [...] (V5, p. 65).

Comme l'avait justement ${ }^{14}$ relevé Yaguello, le livre majeur de Volochinov est «marxiste de bout en bout» (1977, p. 11), et, comme l'ensemble de son œuvre, de ce marxisme cohérent, lucide et courageux que professait également Vygotski. On ne peut dès lors que s'étonner de la légèreté avec laquelle est reproduite l'idée que ce marxisme n'aurait été que « de surface $»^{15}$, et destiné à couvrir une pensée véritable qui serait celle du phénoménologue Bakhtine! Certes, en relisant distraitement des bouts d'écrits de Volochinov avec des lunettes déformantes façonnées dans les textes tardifs de Bakhtine (cf. 4, infra), on peut se construire cette opinion, et, pourquoi pas, affirmer dès lors que «comme Medvedev, Volochinov fait l'effet d'une personnalité relativement terne sur le plan intellectuel» (Depretto, 1997, p. 169); mais toute analyse effective de l'œuvre, dans son contexte, ses objectifs et sa cohérence, suffirait à mettre un terme à ces ragots.

\footnotetext{
13 Volochinov connaissait les travaux de Vygotski, et notamment son article sur la conscience (1925/1994) dont il s'est manifestement inspiré (cf. V1, p. 77 ; V3, p. 108).

${ }^{14}$ Mais à contresens néanmoins, puisque Yaguello attribue clairement la paternité de l'ouvrage à Bakhtine.

${ }^{15}$ Botcharov s'est particulièrement illustré dans ce type d'analyse : «Qu'est-ce que le marxisme dans les textes de Volochinov et Medvedev ? C'est la première couche [...] au fur et à mesure que l'on s'approche du cœur du problème, le poids de la phraséologie marxiste diminue et dans de grandes parties du texte se résume à rien. Un lecteur attentif remarque que la phraséologie obligée ne touche absolument pas le noyau théorique bakhtinien et s'en sépare sans peine » (1993/1997, p. 192). Si Bakhtine, comme Medvedev et bien d'autres, ont cruellement souffert du stalinisme, force est de constater, à la lecture de ce genre de prose, que la perversion intellectuelle qui en est issue continue de produire ses effets.
} 


\section{Les thèmes de Volochinov, et leur reprise-détournement par Bakhtine}

Volochinov a introduit les thèmes majeurs des genres du discours, du dialogisme et de l'attitude responsive active dans le cadre de la problématique générale qui vient d'être résumée; Bakhtine les ayant repris bien plus tard dans Les genres du discours $^{16}$, nous comparerons brièvement le statut qui leur est accordé par les deux auteurs.

S'agissant des genres du discours, Volochinov pose que la diversité des «actes de parole» ou «énonciations» ou encore «modes de discours» n'a «été l'objet d'aucune étude jusqu'à présent ", mais qu'une étude comparative et un classement de ces genres sont absolument nécessaires, dans la mesure où ceux-ci constituent «les formes matérielles précises de l'expression de la psychologie du corps social » (V5, pp. 39-41). Pour lui, il s'agit essentiellement, d'un côté d'étudier la détermination qu'exercent les formes d'organisation politique et les formes d'activités sur ces genres, d'un autre côté de mettre en évidence les différentes formes de représentations collectives que concrétisent les signes organisés dans ces mêmes genres :

Tout signe, nous le savons, résulte d'un consensus entre des individus socialement organisés au cours d'un processus d'interaction. C'est pourquoi les formes du signe sont conditionnées autant par l'organisation sociale desdits individus que par les conditions dans lesquelles l'interaction a lieu. Une modification de ces formes entraine une modification du signe. C'est justement l'une des tâches de la science des idéologies que d'étudier cette évolution sociale du signe linguistique. Seule cette approche peut donner une expression concrète au problème de l'influence mutuelle du signe et de l'être ; c'est seulement à cette condition que le processus de détermination causale du signe apparaitra comme un véritable passage de l'être au signe, comme un processus de réfraction réellement dialectique de l'être dans le signe. (V5, p. 41).

Affirmant à son tour que la problématique de la diversité des genres n'avait jamais été posée (!!! cf. B9, p. 266), Bakhtine souligne d'abord leur infinie variété, découlant elle-même de la "variété virtuellement inépuisable de l'activité humaine». Après avoir substitué à l'approche sociologique de Volochinov la distinction (originale, mais discutable) des "genres premiers » et des "genres seconds », il reformule alors le problème dans sa propre perspective phénoménologique, et ce sous deux angles. Il affirme d'une part que la diversité des genres constitue une sorte de reflet de la personnalité individuelle :

La variété des genres de discours est susceptible de receler la variété des strates et des aspects de la personnalité individuelle, et le style individuel peut instaurer son rapport particulier à la langue commune. (B9, p. 269).

\footnotetext{
16 Comme le relève P. Sériot (communication personnelle), le titre de cet article devrait plutôt être traduit par Les genres de la parole, ou Genres de parole.
} 
Il analyse d'autre part les situations de production verbale en posant l'existence d'un locuteur qui, parmi l'éventail des genres disponibles, procède à des choix « libres », de manière à réaliser son « dessein discursif » singulier :

[...] le dessein discursif du locuteur, sans que celui-ci se départisse de son individualité et de sa subjectivité, s'adapte et s'ajuste au genre choisi, se compose et se développe dans la forme du genre donné. (B9, p. 284).

S'agissant de l'énonciation et du dialogisme qui s'y manifeste, Volochinov souligne bien évidemment leur caractère fondamentalement social :

En réalité, l'acte de parole, ou, plus exactement son produit, l'énonciation, ne peut nullement être considéré comme individuel au sens étroit de ce terme ; il ne peut être expliqué par rapport aux conditions psychophysiologiques du sujet parlant. L'énonciation est de nature sociale. (V5, p. 119).

C'est pourquoi tous les indices de valeur à caractère idéologique, bien que réalisés par la voix des individus (par exemple, dans le mot) ou plus généralement par un organisme individuel, constituent des indices de valeur sociaux, avec des prétentions au consensus social, et c'est seulement au nom de ce consensus qu'ils s'extériorisent dans le matériau idéologique. (V5, p. 42).

Et c'est dans le prolongement de cette approche qu'il développe le thème de l'attitude responsive active ("une compréhension authentique, active, contient déjà l'ébauche d'une réponse ", V5, p. 146), en tant que mécanisme de négociation et d'actualisation des significations (sociales) des signes :

La compréhension est une forme de dialogue; elle est à l'énonciation ce que la réplique est à la réplique dans le dialogue. Comprendre c'est opposer à la parole du locuteur une contre-parole. [...] C'est pourquoi il n'y a pas lieu de dire que la signification appartient au mot en propre. En réalité, celle-ci appartient au mot en tant que trait d'union entre les interlocuteurs, c'est-à-dire qu'elle ne s'actualise que dans le processus de compréhension active, impliquant une réponse. (V5, p. 146).

Bakhtine quant à lui conçoit le dialogue comme un lieu d'expression des positions singulières des interlocuteurs, positions émanant de «l'âme» (cf. B9, p. 301) de ces derniers :

Le dialogue, par sa netteté et sa simplicité, est la forme classique de l'échange verbal. Chaque réplique, aussi brève et fragmentaire soit-elle, possède un achèvement spécifique qui exprime la position $d u$ locuteur - il est possible de répondre, il est possible de prendre, par rapport à cette réplique, une position responsive. (B9, p. 278).

Et dans sa perspective, les signes et autres entités linguistiques n'ont dès lors aucune valeur (sociale ou historique), hors la valeur expressive que leur confère l'intention radicalement singulière de chaque locuteur :

La langue, en tant que système, dispose [...] d'un riche arsenal de moyens linguistiques $[\ldots]$ mais tous ces moyens, en leur qualité de moyens linguistiques, sont 
absolument neutres sur le plan des valeurs du réel. [Un mot] n'est qu'un moyen linguistique virtuel susceptible d'exprimer une attitude émotive-valorielle face au réel et il n'est relaté à aucune réalité déterminée, seul un locuteur peut instaurer cette sorte de relation, c'est-à-dire un jugement de valeur à l'égard du réel, qu'il réalisera à travers un énoncé concret. (B9, p. 291)

Même si nous ne pouvons la prolonger dans l'espace qui nous est imparti, cette étude comparative montre que, jusqu'au bout, Bakhtine a continué d'adhérer à une phénoménologie radicalement dualiste et solipsiste. Dès lors, pour contribuer à l'élaboration d'une science de l'humain accordant aux pratiques langagières la place centrale qui leur revient, en l'occurrence notamment pour poser le problème des conditions d'interaction entre réseaux d'activité humaine et genres de textes (cf. Rastier, 2001) ou celui du rôle de la pratique des genres dans le développement des capacités de pensée et de raisonnement (cf. Bronckart, 2004), c'est à l'œuvre de Volochinov qu'il convient essentiellement de se référer et de puiser, quand bien même il n'y a pas à négliger les apports techniques issus des études proprement littéraires de Bakhtine.

\section{RÉFÉRENCES BIBLIOGRAPHIQUES}

\section{VOLOCHINOV, V.N.}

V1. (1925), « Po tu storonu social'nogo », Zvezda, n 5, pp. 186-214.

(1980), «Au delà du social. Essai sur le freudisme » in Bakhtine, M., Le freudisme, Lausanne, l'Age d'Homme, pp. 32-77.

V2. (1926), «Slovo v jizni i slovo v poézii », Zvezda, nº 6, pp. 244-267.

(1981), «Le discours dans la vie et le discours en poésie » in Todorov, T. (Ed.) Mikhaïl Bakbtine le principe dialogique (pp. 181-215), Paris, Seuil.

V3. (1927), Freïdizm, Moscou-Leningrad, Goslit.

(1980), Le freudisme, Lausanne, l'Age d'Homme.

V4. (1928), « Noveïchie tetchenia lingvistitcheskoï mysli na zapade » (Les courants les plus récents de la pensée linguistique en Occident »), Literatura i marksizm, $n^{\circ} 5$, pp. 115-149.

V5. (1929), Marksizm i filosofia jazykea, Leningrad, Priboï.

(1977), Le marxisme et la philosophie du langage, Paris, Minuit.

V6. (1930), «Stilistika khoudojestvennoï retchi. 1. Tchto takoé yazyk ?» («Qu'est-ce que le langage ? »), Literatournaïa outchioba, $\mathrm{n}^{\circ} 2$, pp. 48-66.

V7. (1930), «Stilistika khoudojestvennoï retchi. 2. Konstrouktsia vyskazyvania », Literatournaïa outchioba, $\mathrm{n}^{\circ}$ 3, pp. 65-87. 
(1981), «La structure de l'énoncé » in Todorov, T. (Ed.) Mikhaïl Bakbtine le principe dialogique (pp. 287-316), Paris, Seuil.

V8. (1930), «Stilistika khoudojestvennoï retchi. 3. Slovo i ego sotsialnaïa founktsia » («Le discours et sa fonction sociale »), Literatournaïa outcbioba, $\mathrm{n}^{\circ}$ 5, pp. 43-59.

V9. (1930), «O granitsakh poetiki i lingvistiki» in $V$ bor'be za marksizm v literatourovedenii, Leningrad, Priboï.

(1981), «Les frontières entre poétique et linguistique » in Todorov, T. (Ed.) Mikbail Bakbtine le principe dialogique (pp. 243-285), Paris, Seuil.

\section{BAKHTINE, M. M.}

B1. (1920-1924 ?), «K filosofii postupka » (manuscrit).

(2003), Pour une philosophie de l'acte, Lausanne, L'Age d'Homme.

B2. (1920-1924 ?), « Avtor i gueroï v estetitcheskö̈ deïatelnosti » (manuscrit).

(1984), «L'auteur et le héros » in Bakhtine, M., Esthétique de la création verbale (pp. 25-210), Paris, Gallimard.

B3. (1924), «Problema soderjania, materiala i formy v slovesnom khoudojestvennom tvortchestve » (manuscrit).

(1978), «Le problème du contenu, du matériau et de la forme dans l'œuvre littéraire ", in Bakhtine, M., Esthétique et théorie du roman (pp. 20-82), Paris, Gallimard.

B4. (1929), Problemy tvortchestva Dostoïevskogo, Leningrad, Priboï.

(1970), La poétique de Dostoïeski, Paris, Seuil.

B5. (1934-1935), «Slovo v romane » (manuscrit).

(1978), « Du discours romanesque » in Bakhtine, M., Esthétique et théorie du roman (pp. 83-233), Paris, Gallimard.

B6. (1937-1938), «Formy vremeni i khronotopa v romane » (manuscrit).

(1978), «Formes du temps et du chronotope dans le roman» in Bakhtine, M., Esthétique et théorie du roman (pp. 235-398), Paris, Gallimard.

B7. (1941 ?), « K filosofskim osnovam goumanitarnykh naouk » (manuscrit).

(1984), «Remarques sur l'épistémologie des sciences humaines » in Bakhtine, M., Esthétique de la création verbale (pp. 379-393), Paris, Gallimard.

B8. (1946), Tvortchestvo Fransoua Rable i narodnä̈a koultura srednevekovia $i$ Renessansa (manuscrit).

(1970), L'auvre de François Rabelais et la culture populaire au moyen âge et sous la renaissance, Paris, Gallimard.

B9. (1952-1953), «Problema retchevykh janrov » (manuscrit). 
(1984), «Les genres du discours » in Bakhtine, M., Esthétique de la création verbale (pp. 263-308), Paris, Gallimard.

B10. (1959-1961), « Problema teksta v lingvistike, filologuii i drouguikh goumanitarnykh naukakh. Opyt filosofskogo analiza » (manuscrit).

(1984), «Le problème du texte » in Bakhtine, M., Esthétique de la création verbale (pp. 309-338), Paris, Gallimard.

\section{Autres références}

Aucouturier, M. (1978), «Préface » in Bakhtine, M., Esthétique et théorie du roman, Paris, Gallimard, pp. 9-19.

Botcharov, S.G. (1997[1993]), «A propos d'une conversation et autour d'elle » in Depretto, C., L’héritage de Bakbtine, Bordeaux, Presses universitaires, pp. 180-204.

Brentano, F. (1944[1874]), La psychologie d'un point de vne empirique, Paris, Aubier.

BRONCKART, J.-P. (2004), «La médiation langagière, son statut et ses niveaux de réalisation » in Delamotte-Legrand, R. (Ed.), Les médiations langagières. Vol. II, Des discours aux acteurs sociaux, Rouen, PUR, pp. 11-32.

BRONCKART, J.-P. \& FriedRICH, J. (1999), «Présentation» in Vygotski, L.S., La signification bistorique de la crise en psychologie, Paris, Delachaux et Niestlé, pp. 15-69.

BÜHLER, K. (1927), Die Krise der Psychologie, Jena, Fischer.

Deprettto, C. (Ed.) (1997), L'héritage de Bakbtine, Bordeaux, Presses universitaires.

DEWEY, J. (1925), Experience and Nature, New York, Dover.

DURKHEIM, E. (1951), «Représentations individuelles et représentations collectives », in Sociologie et philosophie, Paris, PUF, pp. 1-48 [initialement publié dans la Revue de métaphysique et de morale, 1898, nº 6, pp. 273-302].

ENGELS, F. (1975[1925]), Dialectique de la nature, Paris, Editions sociales.

Husserl, E. (1931), Méditations cartésiennes, Paris, Vrin.

IVANOVA, I. (2003a), «Le dialogue dans la linguistique soviétique des années 19201930 » in Sériot, P. (Ed.), Le discours sur la langue en URSS à l'époque stalinienne, Cabiers de l'Institut de linguistique et des sciences du langage, $\mathrm{n}^{\circ}$ 14, pp. 157-182.

IVANOVA, I. (2003b), «Les sources de la conception du dialogue chez L. Jakubinskij », Texto!, décembre 2003 [en ligne]. http://www.revue-texto.net/Inedits/Ivanova Jakubinskij.html.

JAKUBINSKIJ, L. (1923), «O dialoguitcheskoï rechi » («Sur la parole dialogale ») in Russkaja Rech', n 1.

KristeVA, J. (1970), «Une poétique ruinée » in Bakhtine, M., La poétique de Dostoïevskei, Paris, Seuil, pp. 5-27. 
MARX, K. (1951[manuscrit rédigé en 1845]), «Thèses sur Feuerbach » in Marx, K. \& Engels, F., Etudes philosophiques, Paris, Editions sociales, pp. 61-64.

MEAD, G.H. (2006[1934]), L'esprit, le soi et la société, Paris, PUF.

Politzer, G. (1928), Critique des fondements de la psychologie, Paris, Rieder.

POTEBNYA, A. A. (1913), Mysl' i yasyk, Kiev, SINTO.

Rastier, F. (2001), Arts et sciences du texte, Paris, PUF.

SÉRIOT, P. (2007), « Histoire des idées linguistiques. Le monde de N. Volochinov. Le néo-humboldtianisme en Russie actuelle » in Annuaire des cours, Faculté des Lettres, Université de Lausanne, http://www.unil.ch/slav/ling/cours.

Todorov, T. (Ed.) (1981), Mikbail Bakbtine le principe dialogique, Paris, Seuil.

VYGOTSKI, L.S. (1994[1925]), « La conscience comme problème de la psychologie du comportement », Société française, $\mathrm{n}^{\circ}$ 50, pp. 35-50.

VyGotSKi, L.S. (1997[1934]), Pensée et langage, Paris, La Dispute.

VYGOTSKI, L.S. (1999[manuscrit rédigé en 1927]), La signification historique de la crise en psychologie, Paris, Delachaux et Niestlé.

WundT, W. (1874-1875), Grundrüge der physiologischen Psychologie, Leipzig, Engelmann.

WunDT, W. (1900-1920), Völkerpsychologie, Bd. 1 - 10, Leipzig, Engelmann.

YAguelLo, M. (1977), «Introduction » in Bakhtine, M., Le marxisme et la philosophie du langage, Paris, Minuit, pp. 9-18. 
